\title{
Design and Development of Medical Image Diagnosis Report System
}

\author{
Bing-jin Liang ${ }^{a}$, Hao-yu Jin $^{a^{*}}$, Guo-dong Liu ${ }^{b}$ Xiao-mian $\mathrm{Ma}^{a}$ \\ ${ }^{\mathrm{a}}$ Guangdong Food and Drug Vocational College, Guangdong, China \\ ${ }^{\text {b} G u a n g z h o u ~ E l e c t r o n i c ~ G o v e r n m e n t ~ A f f a i r s ~ S e r v i c e ~ C e n t e r, ~ G u a n g d o n g, ~ C h i n a ~}$ \\ *Corresponding author: Hao-yu Jin, Ph.D, jinhy@gdyzy.edu.cn
}

\begin{abstract}
The purpose of this paper is to develop an efficient medical image report system, which is consistent with the printing effect, display, editing and other interface effects. In the project, we instantiate the report items as a text box control, drop-down box control, image control, etc. These controls are distinguished by different parameters, and use XML to format the control parameters and save to an Oracle database. The system is applied to the Department of Radiology, Endoscopy, Ultrasound and other departments in the hospital. Diagnosis doctors can directly edit reports on the visual interface, including text editing, changes image and other operations. It can be found that the report system shows the same effect as the print effect. The system solves the problem of consistency between display and print, which greatly facilitates the preparation of the report. The final use of the project achieves the purpose of design.
\end{abstract}

Key words: WYSIWYG; medical diagnostic report; XML; medical system

\section{Introduction}

With the development of computer technology, hospital information has been popularized and deepened. The popularization of hospital information technology has greatly improved the efficiency of medical staff and improved the overall efficiency of the hospital from the traditional manual process to the digital process of hospital computer. Hospital information system has experienced from the medical staff to solve the problem of information entry, how to improve the diagnostic efficiency and accuracy of diagnosis. ${ }^{1,2}$

The diagnostic report is an important part of the Radiology Information System (RIS), ${ }^{3}$ Ultrasonography Information System (UIS), ${ }^{4,5}$ and Endoscopy Information System (EIS) ${ }^{6,7}$ and so on. From the perspective of the diagnosis of doctors, they hope that the system is easy to use and shown the same effect as the print. From the perspective of Information Department, they hope that the report format has readability and easy interactive features, and the report data are easy exchanging among different information systems in hospital. From the perspective of the medical system company, they hope that the report format can be unified to reduce the difficulty of implementation and shorten the implementation cycle.

At present, some scholars have done some research; there are some third party tools, such as through the VB programming, Word mode, report software and so on. But the integration of 
these methods and systems is not high, and is not convenient to use. Especially the problem of third party plug-in is easy to cause the instability of the system. Therefore, this paper describes a WYSIWYG ${ }^{8,9}$ report system developed by the Microsoft VC++ development tool. $^{10}$

\section{Architecture design}

\subsection{Report process}

Medical diagnostic reports are completed by different levels of doctors. The report flow chart is shown below (Fig.1).

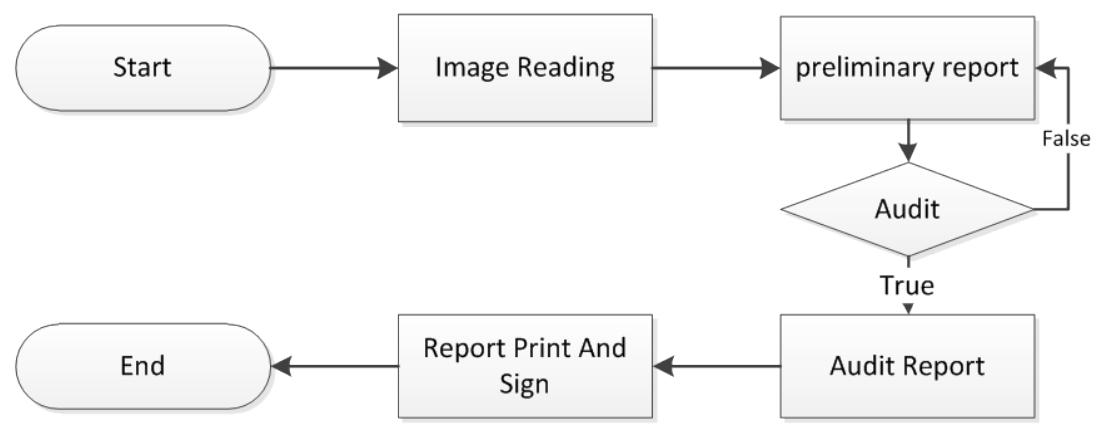

Fig.1 Report flow chart

Diagnosis doctor opens patient's image to read the film, and writes the preliminary report by using diagnostic workstation. While the preliminary report was submitted, the upper doctor will check and audit report. If the audit is not passed, the report will be sent back to the preliminary report doctor to be rewritten; if it is a small problem only minor modifications, the higher level doctors can directly modify it. The report will be audited, printed and signed after be modified.

Consider editing, printing, clinical browsing and other functions, the systems are divided into three parts: report pattern editor, diagnostic report writing system and clinical browsing system. Using the template editor, user generates report template; using the report system, report template be loaded and report be written; the clinician through the clinical workstation could view the audit report.

\subsection{Report element design}

In the medical diagnostic report, there are static text boxes, editable text box, split line, dropdown list, check box, medical image control, barcode control and so on. In the architecture design, we instantiate the relate elements. According to the role of the control, classes like CReportLabel, CReportEdit, CReportLine, CReportCombobox, CReportCheckBox, CReportImage, CReportBarCode are created. Public properties are extracted to class CReportEntity. Also, we create CReportPageContainer to manage CReportPage for report paging. The report system class diagram as shown below (Fig.2). The classes of controls are shown in table 1. 


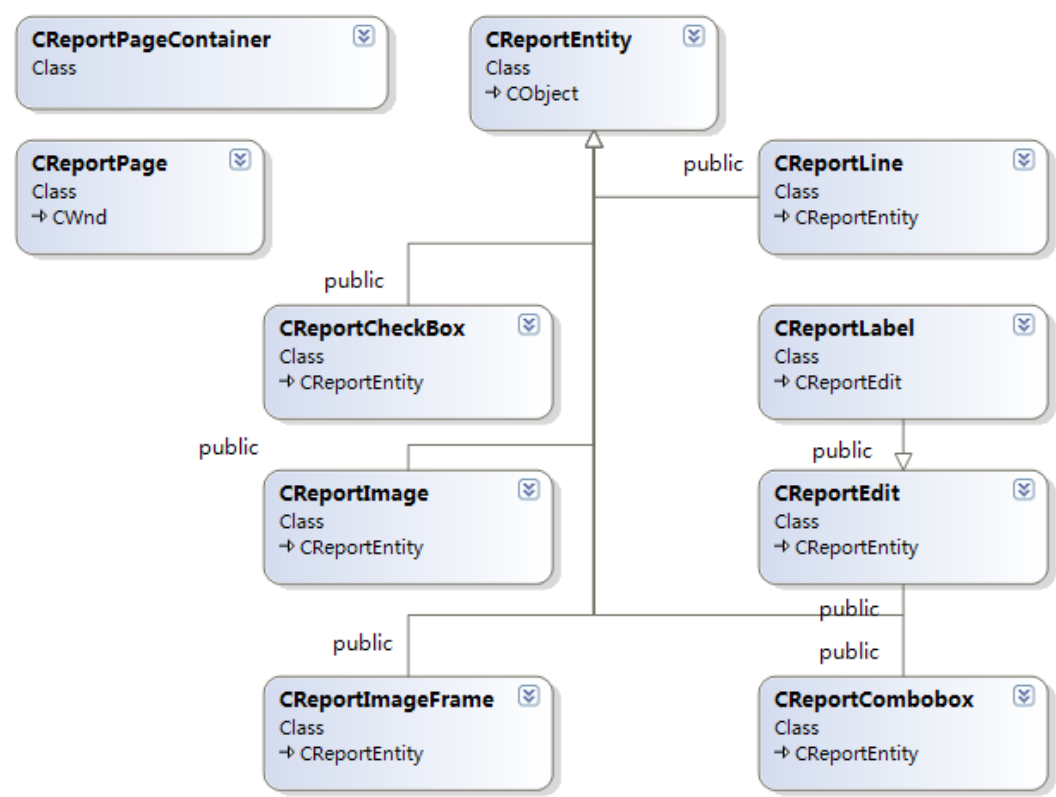

Fig. 2 System Class Diagram

Table 1 The Class Of Controls Description

\begin{tabular}{|l|l|}
\hline The Class Of Controls & Description \\
\hline CReportLabel & Uses to display uneditable text, like name, sex, birthday etc.. \\
\hline CReportEdit & User can input report content in it \\
\hline CReportLine & Line, Uses to separate controls \\
\hline CReportCombobox & Drop down list control \\
\hline CReportCheckBox & Check box control \\
\hline CReportImage & Display exam image, hospital logo \\
\hline CReportBarCode & Display barcode information, the content may be 'patientid' \\
\hline OtherControls & \\
\hline
\end{tabular}

\subsection{Report Element Storage}

$\mathrm{XML}^{11-13}$ has the advantages of flexibility, hierarchy and so on. Therefore, we use the XML format to save the diagnostic report to database. In this project, we use Oracle database and the XML data are saved into BLOB type field. When press the 'Save' button, the system will call the 'SaveXML' function to save the report data like the location of the elements, the current value, font content, image path, bar code, and so on. In order to reduce the size of XML data, the system compress the name of the node when the relevant nodes are stored such as the name of the [NAME], only [N]. As shown in table 2, we illustrate the properties of the classes and the storage nodes. 


\section{System usages}

\subsection{Implement engineer create report pattern}

In order to speed up the creation of the report pattern, the project team developed pattern editor software. The user of the pattern editor software is the administrator of the reporting system or the project implement engineer. By using the software, we can easily complete the creation, modification and preservation of the report pattern on the visual interface. The interface of the software is shown below (Fig.3).

Table 2 Class Property and Description

\begin{tabular}{|l|l|l|}
\hline FullName & ShortName & Description \\
\hline Name & N & Control name, unique number \\
\hline Type & TY & Control type, text box, drop-down box, etc. \\
\hline TabStop & TS & Control's tabIndex property \\
\hline PageIndex & PI & Control's tabStop property \\
\hline Position & PS & The page index of the control locate \\
\hline FontFormat & FM & The position of the control locate \\
\hline Text & T & The font color, font size etc.. \\
\hline OtherNames & & The text value of the control \\
\hline
\end{tabular}

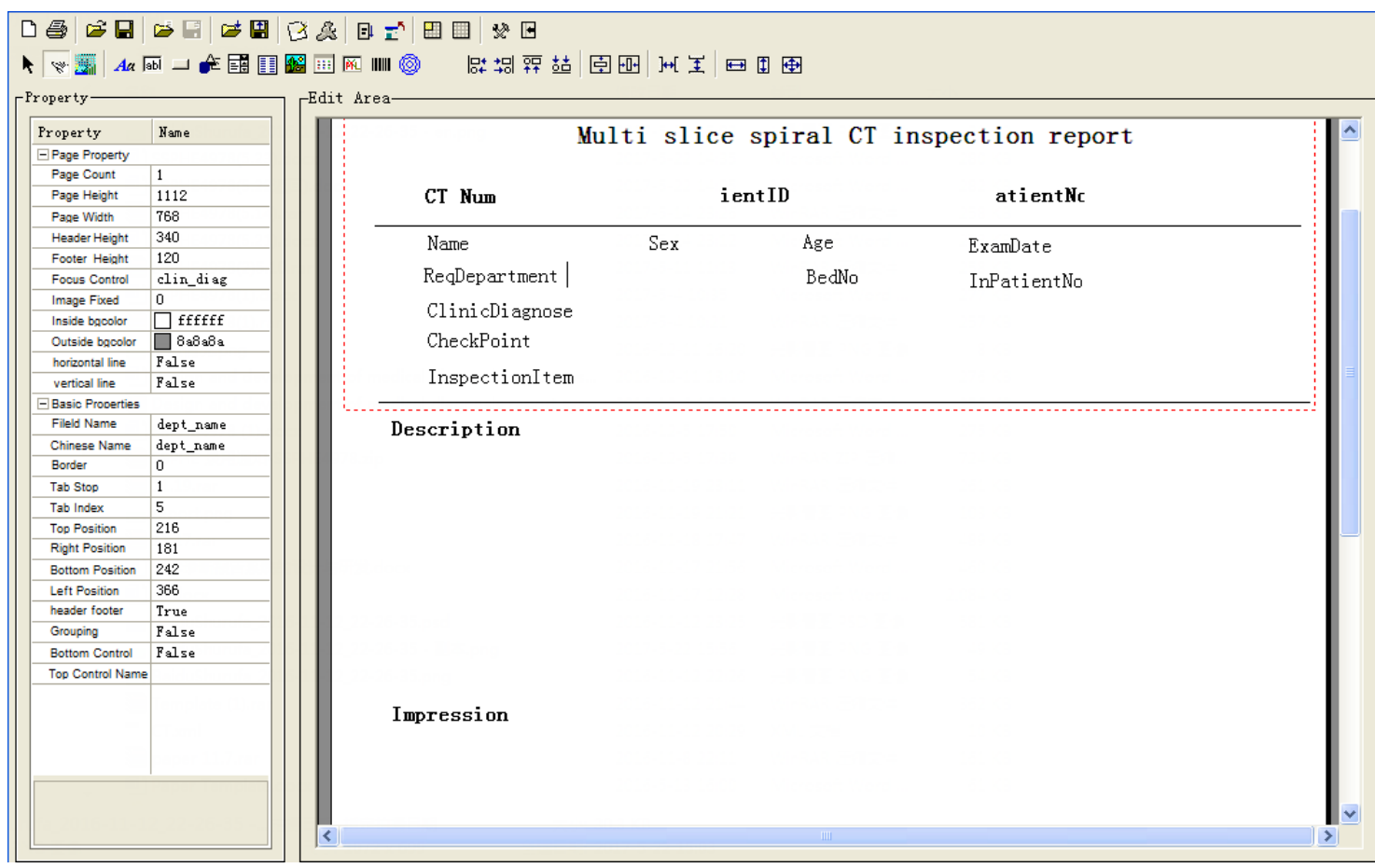

Fig. 3 report pattern software

(1) Control create

Controls will be created when click on the toolbar button. For example, if we want to create 
a 'edit control', click on the appropriate button, press the left button in the report edit area and drag, an edit control will be created with a certain size.

(2) Property setup

When the mouse clicks on the control, the control is selected. The control's properties will show on the left area. We can change the values on property boxes like name, border, and position and so on.

(3) Control saves

While all the controls have been created, the report pattern should be saved with the click on the 'save' button. There are two methods to save the pattern: XML file or database. If saved as a XML file, the XML content is stored directly to the specified file. If saved to the database, we will save the XML content to the database of the large data fields in the BLOB field.

\subsection{Doctor write diagnose report}

After getting the patient application form, doctor searches the patient's examination information in the query system, selects inspection records and opens the reporting system to prepare the report. When writing a report for the first time, the system will judge the type of inspection or other information, and load the report pattern which is done by the report pattern editor. After that, the system automatically fills the patient's name, the date of inspection, the date of report and other information on the empty report pattern.

When the report has been initialized, doctor mainly input the "impression" and" impress" controls' content. In order to speed up the content editing, we collect the common use report terms and form the diagnosis knowledge library. By using diagnosis knowledge library, doctor can quickly fill the item values into report, modify some values and save it. These greatly accelerate the report writing speed.

While the report has been competed, we can click on the toolbar's 'save' button, or press 'Ctrl + S' shortcut key to save the report. The report controls, control's values and other parameters are reformed to XML file and saved to the database's report table.

The report status is 'preliminary report' while first saved, and submitted to upper level doctor to be audited. The upper level doctor modifies or audits the preliminary report and the report status changed to 'audit report'. Now, the report can be printed and sent to patient.

\subsection{Clinical report browsing}

There are great many and complex clients in the clinical system to browse reports. In order to adapt to the desktop, mobile terminal and other applications, we use the WEB way to view the reports. Clinical WEB report browsing system is developed by ASP.NET and jQuery. JQuery library is a cross platform Javascript processing library. By using the jQuery, we can quickly carry out the development of the web page. The system uses ASP.NET to query the database, get the patient's XML report, sends to the front-end page, parser and displays by using jQuery. We can also use the clinic system to print the patient report if needed. 


\section{System implementation result}

This report system is developed by $\mathrm{C}++$ program language and its database is Oracle. $\mathrm{C}++$ connects with Oracle database by using ADO. The report system effect is shown below (Fig.4). Like the name of the hospital, report name, patient name and other fields are not allowed to edit. These fields can be used in a static text box. In the medical image fields, you can use the medical image box; in "description”, “impress” parts, you can use editable text box. When report is opened, these objects will be initializing and filled. The initial data read from Oracle database, which reduces the amount of input information to facilitate the doctor to prepare a report.

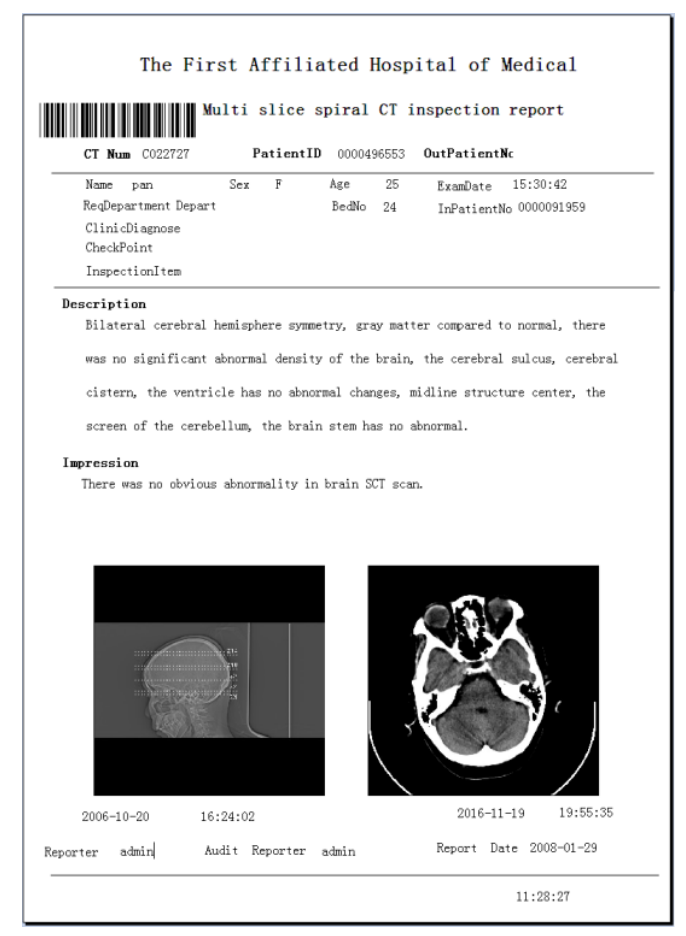

Fig. 4 Report system effect

\section{Conclusions}

By using the report system, doctor writes diagnosis report in print like interface, and the report printing effects and editing results are consistent. To sum up, the system has the following advantages:

(1) The diagnosis report system is 'what you see is what you get' report system. The effect of editing interface is consistent with the printing effect, intuitive and clear.

(2) The report is saved with XML format which is easy to retrieve, store and format display.

(3) The report administrator can quickly make a report pattern by using report pattern editor. This greatly speeds up the progress of the project implementation.

The system was first used in the radiology department, and then extended to endoscopy department, pathology department and other departments. At present, the system is running in a number of large hospitals. Practice has proved that the system has improved the efficiency 
of the doctor writing report, and is well received by the medical staff.

\section{Acknowledgements}

This work is supported by GDHVPS(2016), Medical Scientific Research Foundation of Guangdong Province (No: A2016044), Science and Technology Planning Project of Guangdong Province (No: 2016A020216016) and the Fundamental Research Funds of Guangdong Food and Drug Vocational College (No: 2015YZ017).

\section{References}

1. P. L.Reichertz, Hospital information systems--past, present, future, International Journal of Medical Informatics, 2006, 75(4):282-299.

2. G.White, R. Hill, M. J. Zakrewski, et al., Patient medication IV delivery pump with wireless communication to a hospital information management system: US, US 8486019 B2, 2013.

3. A.Rumballsmith, S. Macdonald, Development and Utilisation of a Real-Time Display of Logged in Radiology Information System Users, Journal of Digital Imaging, 2011, 24(2):295-9.

4. J. B.Seward, B. K. Khandheria, J. A. Koerner, et al., Ultrasound laboratory information management system and method, 2013.

5. S.Lin, Z.Yu, Ultrasound information processing system: US6547730,2003.

6. N. K.Besedina, A. V. Kuznetsov, The Evis-"Elektron" endoscopic video-information system, Biomedical Engineering, 2003, 37(3):161-162.

7. H.Yokoi, M. A.Fujino, Activities for Endoscopy Information Systems Standardization in Japan,2006:5667-5670.

8. L.Yin, J. An, X. Lu, et al. A WYSIWYG template designer for medical report systems,Biomedical Engineering and Informatics (BMEI), 2012 5th International Conference on. IEEE, 2012: 1035-1039.

9. O.Yanar, O. E. Balcicek, Interactive reporting architecture: A WYSIWYG approach to enterprise reporting, Technological Advances in Electrical, Electronics and Computer Engineering (TAEECE), 2013 International Conference on. IEEE, 2013: 387-393.

10. Z. F.Yi, J. Sun, Y. Y. Huang, et al. Automatic Calibration of Temperature Sensor System Base on Visual C++, Energy Procedia, 2011, 13:8963-8968.

11. H.Liu, C. Friedman, A method for vocabulary development and visualization based on medical language processing and XML, Proceedings / AMIA. Annual Symposium, AMIA Symposium, 2000:502-6.

12. H. B.Bludau, A.Wolff, A. J. Hochlehnert, Presenting XML-based medical discharge letters according to CDA, Methods of Information in Medicine, 2003, 42(42):552-6.

13. M. M.Triola, N. Campion, J. B. Mcgee, et al., An XML Standard for Virtual Patients: Exchanging Case-Based Simulations in Medical Education,AMIA, Annual Symposium proceedings / AMIA Symposium, AMIA Symposium, 2007, 2007:741-5. 